Erratum

\title{
Erratum to "Tumor Hemodynamics and Hepatocarcinogenesis: Radio-Pathological Correlations and Outcomes of Carcinogenic Hepatocyte Nodules"
}

\author{
Kazuhiko Ueda, ${ }^{1}$ Osamu Matsui, ${ }^{2}$ Azusa Kitao, ${ }^{2}$ Satoshi Kobayashi, ${ }^{2}$ \\ Jun Nakayama, ${ }^{3}$ Shinich Miyagawa, ${ }^{4}$ and Masumi Kadoya ${ }^{1}$ \\ ${ }^{1}$ Department of Radiology, Shinshu University School of Medicine, 3-1-1 Asahi, Matsumoto 390-8621, Japan \\ ${ }^{2}$ Department of Radiology, School of Medicine Kanazawa University, 13-1 Takara-machi, Kanazawa 920-8641, Japan \\ ${ }^{3}$ Department of Molecular Pathology, Shinshu University School of Medicine, 3-1-1 Asahi, Matsumoto 390-8621, Japan \\ ${ }^{4}$ Department of Surgery, Shinshu University School of Medicine, 3-1-1 Asahi, Matsumoto 390-8621, Japan
}

Correspondence should be addressed to Kazuhiko Ueda; uedakazu@shinshu-u.ac.jp

Received 24 July 2014; Accepted 7 August 2014; Published 21 October 2014

Copyright (c) 2014 Kazuhiko Ueda et al. This is an open access article distributed under the Creative Commons Attribution License, which permits unrestricted use, distribution, and reproduction in any medium, provided the original work is properly cited.

In the paper entitled "Tumor hemodynamics and hepatocarcinogenesis: radio-pathological correlations and outcomes of carcinogenic hepatocyte nodules," Page 8, Figure 12, figure legends, first sentence, line 2 should be corrected as follows: "without capsule" should read "with capsule." 


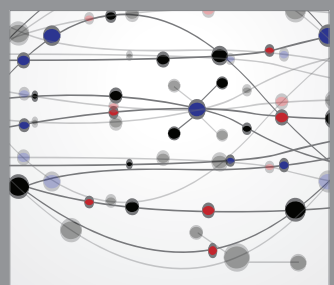

The Scientific World Journal
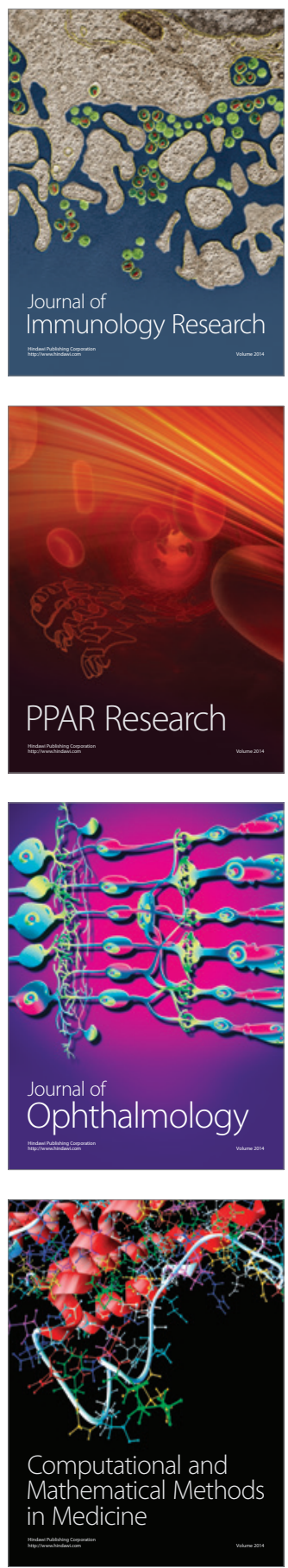

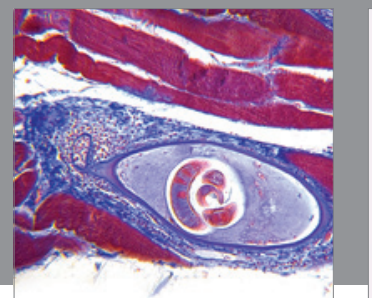

Gastroenterology

Research and Practice
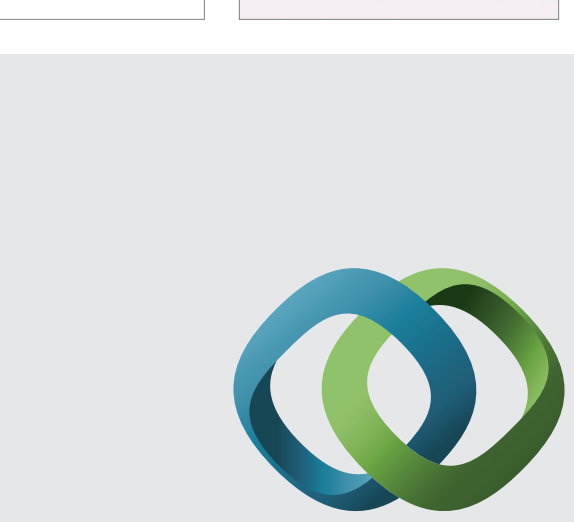

\section{Hindawi}

Submit your manuscripts at

http://www.hindawi.com
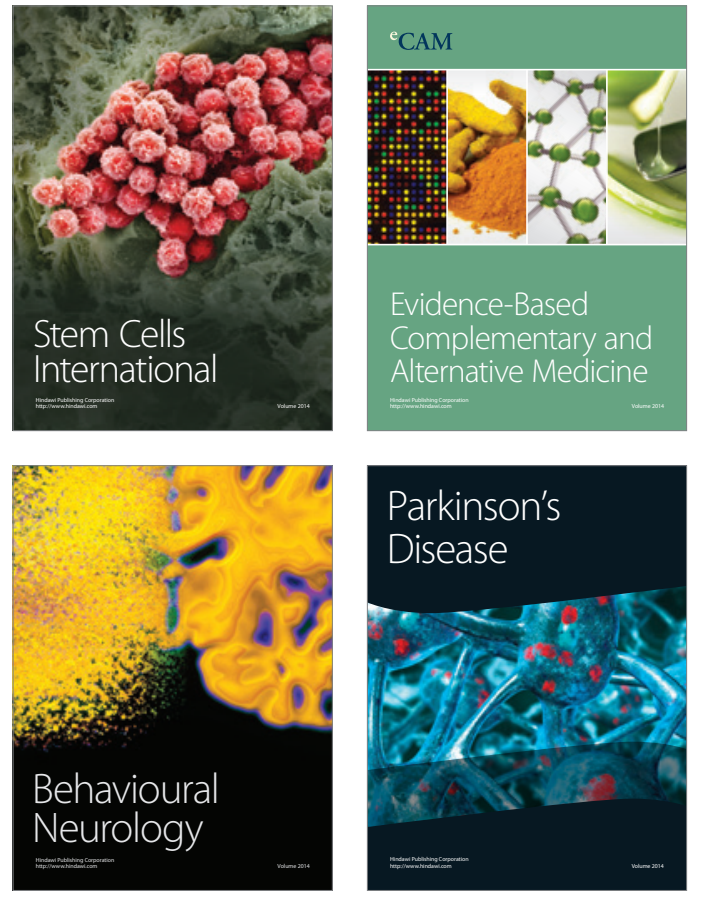
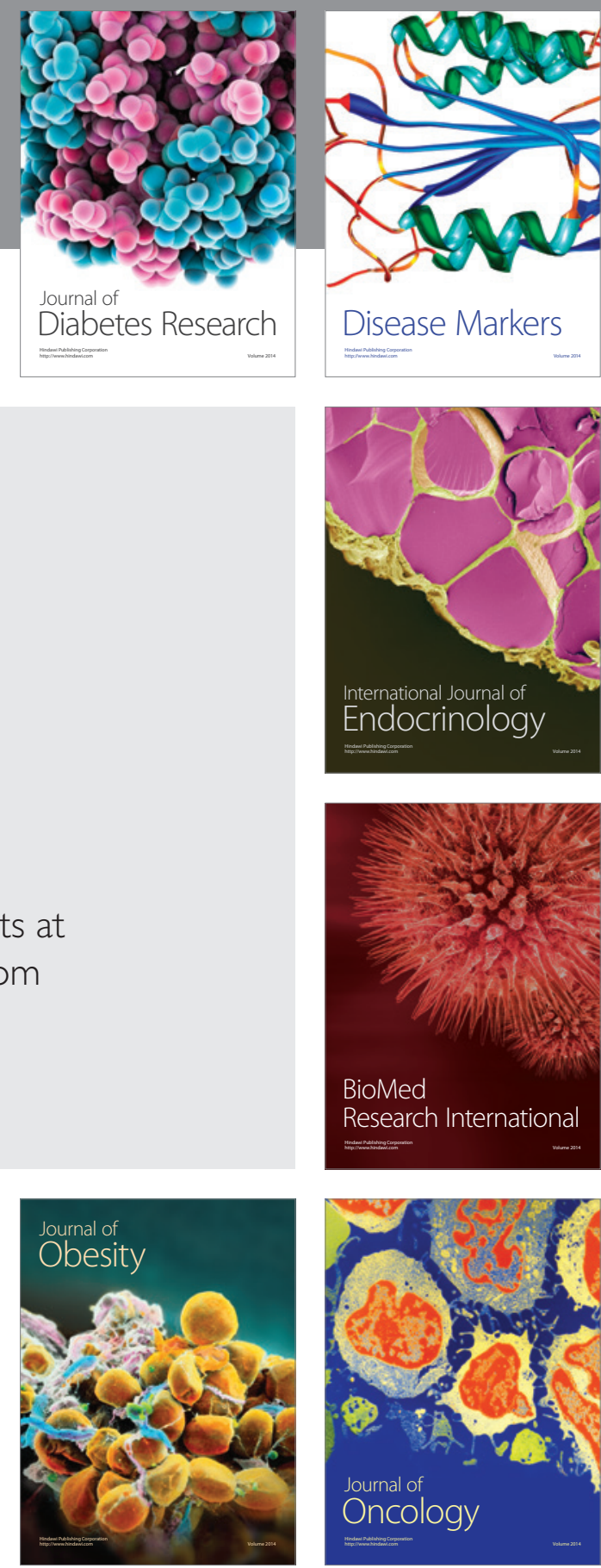

Disease Markers
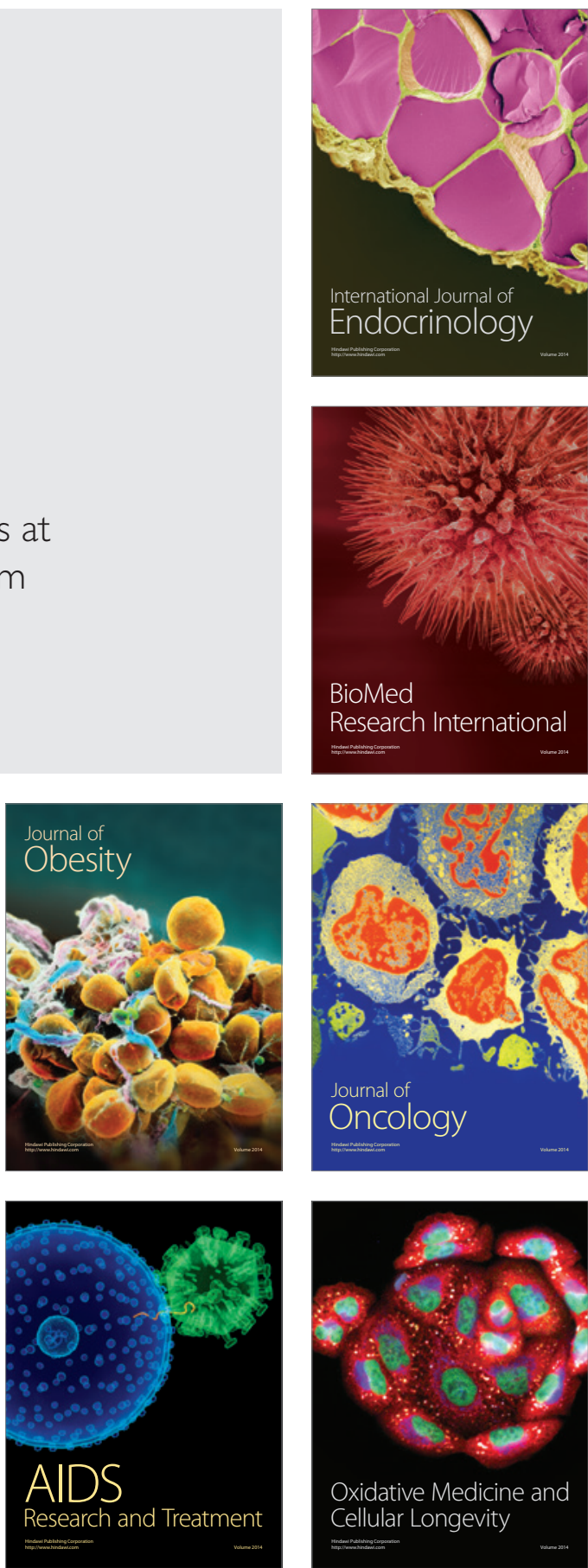